\title{
LA ADOLESCENCIA: AÑEJOS DEBATES Y CONTEMPORÁNEAS REALIDADES
}

\author{
IDIANELYS SANTILLANO CÁRDENAS ${ }^{*}$
}

\begin{abstract}
RESUMEN
El análisis de la adolescencia como etapa supone adentrarse en referencias teóricas que históricamente han estado en debate, y sentar posición acerca de las mismas con una mirada más actualizada. Ello enriquece cualquier análisis posterior y ayuda a una mejor comprensión de algunos elementos asociados a las circunstancias actuales en la que se vive la adolescencia hoy día. Sobre estos temas específicamente trata el artículo, con la intención de abrir interrogantes e incentivar quehaceres a favor de un mañana mejor, sobre la base de una adolescencia, hoy, con mayores posibilidades de tener un contexto de desarrollo más favorable.
\end{abstract} PALABRAS ClAVE: DESARROLLO PSICOLÓGICO,
SOCIEDAD ACTUAL, ADOLESCENTES

* Licenciada en Psicología, Diplomada en Salud Sexual y Reproductiva del Adolescente. Actualmente cursa la maestría en Metodología de los Procesos Correctores Comunitarios. Investigadora del Centro de Estudios Sobre la Juventud y se desempeña como jefa del Departamento Sociocultural. E-mail: dcesj@jovenclub.cu. 


\title{
A ADOLESCÊNCIA: ANTIGOS DEBATES E REALIDADES CONTEMPORÂNEAS
}

\begin{abstract}
RESUMO
A análise da adolescência como etapa requer a busca de referências teóricas, que historicamente têm estado em discussão, bem como o estabelecimento de uma posição sobre as mesmas com um olhar mais atualizado. Isto enriquece qualquer análise posterior e auxilia numa melhor compreensão de alguns elementos associados às circunstâncias atuais da adolescência hoje em dia. $\mathrm{O}$ artigo trata destes temas especificamente com o propósito de propor questionamentos e incentivar ações a favor de um futuro melhor, sobre a base de uma adolescência que hoje conta com maiores possibilidades de um contexto de desenvolvimento mais favorável.
\end{abstract}

\section{PALAVRAS-CHAVE: DESENVOLVIMENTO PSICOLÓGICO, SOCIEDADE ATUAL, ADOLESCENTES}

\section{ADOLESCENCE: ANNEX DEBATES AND CONTEMPORARY REALITIES}

\begin{abstract}
The analysis of adolescence as a stage is go into theoretical references that have historically been under discussion, and lay about the same position with a more updated look. This enriches any further analysis and helps a better understanding of some elements associated with the current circumstances in which we live today adolescence. On these issues specifically in article, intended to raise questions and encourage chores for a better tomorrow, on the basis of a teenager today, most likely to have a more favorable development context.
\end{abstract}

KEY WORDS: PYCHCOLOGICAL DEVELOPMENTS, CURRENT SOCIETY, ADOLESCENTS 


\section{Algunas CUESTIONES REFERIDAS A LA CONCEPTUALIZACIÓN DE LA ADOLESCENCIA}

«ATRAPAR» LA ADOLESCENCIA DESDE las ciencias sociales, se ha convertido en una tarea tan compleja, como lo es - en ocasionesentender a quienes están en esa etapa de la vida. Los primeros intentos se localizan a finales del siglo XIX y principios del XX, cuando el psicoanalista Stanley Hall publicó sus trabajos sobre el tema. Desde entonces la historia recoge diversos modos de acercamiento a ese periodo, los cuales han sido más o menos compartidos por la comunidad científica, en la medida que el propio desarrollo de las ciencias ha ofrecido argumentos y ha consensuado tendencias.

Una de las polémicas principales se centra en los enfoques referidos a la caracterización de la adolescencia. El investigador ex soviético Kon definió tres: el biogenético, el sociogenético y el psicogenético (Kon, en Domínguez, 2003); los cuales también fueron concebidos con otra nomenclatura por el científico Juan Deval (Deval, en Dávila et al., 2008). Este último se refirió puntualmente a la teoría psicoanalítica, la sociológica y la de Piaget; sin embargo, al indagar en sus contenidos, estos pueden hacerse corresponder respectivamente con los expuestos por Kon, de acuerdo con la esencia que postulan.

Los tres enfoques acerca de la adolescencia tienen como denominador común el hecho de enfatizar en alguna de las condicionantes del desarrollo, mientras que difieren en la preponderancia conferida a uno $\mathrm{u}$ otro respecto al resto. Por un lado, el biogenético concibe lo biológico como el principal determinante del desarrollo psíquico, de ahí que en primera instancia considere primordial verificar la maduración del adolescente desde este punto de vista.

Por otra parte, el sociogenético distingue, sobre todo, las regularidades que tienen lugar en el proceso de socialización, vinculadas a la actividad. De esta forma, supone que las particularidades del entorno pasan a desempeñar un papel básico, y el planteamiento de tareas por parte de la sociedad marca sustancialmente el desarrollo de los procesos psíquicos. Si bien ambas miradas consideran la relevancia que tiene lo extrapsíquico - hecho que también reconoce el enfoque psicogenético-, este último centra su atención en las funciones y procesos psicológicos que caracterizan al periodo, con lo cual tiene el mérito de girar la mirada al interior del sujeto (Domínguez, 2003).

Desde nuestro punto de vista, es sumamente importante la integración de los elementos sociales, psicológicos y biológicos, pues 
todos ellos sufren grandes cambios en esta etapa y cada adolescente es resultado de la forma en que se combinan para su caso particular. Sin embargo, compartimos sobre todo la concepción de la adolescencia como construcción sociohistórica (Obiols, 1995; Dávila et al., 2008; Martínez, 2009), cuya implicación teórica, sitúa el análisis de la categoría, a partir de lo cultural y lo relacional en las sociedades contemporáneas, más allá de lo biológico.

Considerando esta perspectiva, concebimos la adolescencia como resultante de «construcciones y significaciones sociales en contextos históricos y sociedades determinadas, en un proceso de permanente cambio y resignificaciones» (Dávila et al., 2008:43); y aunque parecería que lo biológico queda a la zaga, en realidad, el desarrollo físico - entiéndase cambios puberales - es asumido como un proceso concomitante al fenómeno sociopsicológico que es la adolescencia en sí misma.

Estas distinciones permiten asumir una posición diferente no sólo ante la adolescencia como categoría de las ciencias, sino también respecto al sujeto adolescente; a ése que muchas veces se observa como parte de una generalidad y dentro de un esquema de regularidades etáreas, como si de manera inamovible tuviera que ser de esa forma y no de otra. Paralelamente, esta concepción que enfatiza en lo social, ofrece la posibilidad de romper con el tradicional término biopsicosocial, para legitimar el sociopsicobio, confiriéndoles a cada arista el lugar verdadero que posee en la construcción de la psiquis humana, de acuerdo con nuestra concepción.

Debe señalarse que la comprensión sociohistórica no implica un sin fin de incertidumbres ante qué es ser adolescente, pues existen muchas certezas al respecto. En todo caso deja claro que lo visto hoy en las familias, en las escuelas, en las ciudades, no sólo posee algunas diferencias respecto a los adolescentes de los 60, por citar un ejemplo, sino que prácticamente no tiene que ver con quienes compartían esa edad siglos atrás, aunque los cambios puberales eran los mismos. Parecería obvio, pero algunos análisis insuficientemente contextualizados acerca de cómo crecen hoy los y las adolescentes, muestran lo poco que se toma en consideración esta perspectiva histórica tanto en las investigaciones, como en las valoraciones empíricas que en ocasiones se hacen, de quienes están viviendo ese periodo de sus vidas.

Además de los enfoques en torno a la caracterización de la etapa, otra cuestión que ha generado arduo debate ha sido el establecimiento de los límites etáreos en los cuales enmarcarla. Convencionalmente se 
ha utilizado el rango entre los 12 y los 18 años (Dávila et al., 2008), pero las Organizaciones Mundial y Panamericana de la Salud la ubican entre los 10 y los 19 (2002), el psicoanálisis freudiano entre los 12 y los 15 (Domínguez, 2003) y la autora Bozhovich (1976) considera que la etapa va de los 11 ó 12 años a los 14, aproximadamente, y la nombra edad escolar media. Todo ello muestra la diversidad de criterios existentes y, al mismo tiempo, evidencia la coincidencia de ubicación en la segunda década de la vida.

Al parecer, cada vez más carece de importancia pautar estrictamente el comienzo y el final. En su lugar se ha preferido esbozar con relativa flexibilidad sus límites y considerar, principalmente, el nivel de desarrollo integral alcanzado por la persona para su edad cronológica. No obstante, el consenso mayor está entre el comienzo de los cambios puberales (alrededor de los 10 años) y el tiempo en que se visualizan más o menos consolidadas dichas transformaciones; unido a las correspondientes modificaciones en las esferas social y psicológica (alrededor de los 19 ó 20 años).

Por último, existe un tercer tema que ha suscitado numerosos análisis: la definición de adolescencia. El ya mencionado psicólogo norteamericano Stanley Hall, pionero en los estudios sobre adolescencia, la consideraba «una edad especialmente dramática y tormentosa en la que se producen innumerables tensiones, con inestabilidad, entusiasmo y pasión [...], supone un corte profundo con la infancia, es como un nuevo nacimiento en el que el joven adquiere los caracteres humanos más elevados» (Hall, en Dávila et al., 2008:46).

Conceptualmente, Stanley distingue los conflictos que tienen lugar en la etapa, su posición posterior a la infancia y la obtención de nuevas características que preparan para posteriores periodos, elementos estos que otros autores también han tomado en consideración (Domínguez, 2003; Gutiérrez, 2007; Dávila et al., 2008).

Si bien nadie niega la importancia de las transformaciones que ocurren, algunos investigadores enfatizan más en lo psicológico y otros en lo social. Un ejemplo de ello es la conceptualización de la adolescencia como «etapa de la vida durante la cual el individuo busca establecer su identidad adulta, apoyándose en las primeras relaciones objetales/parentales internalizadas y verificando la realidad que el medio le ofrece, mediante el uso de los elementos biofísicos en desarrollo a su disposición y que a su vez tienden a la estabilidad de la personalidad en un plano genital, lo que sólo es posible si se hace el duelo por la identidad infantil» (Knobel, 2008:5). Esta idea tomada del 
psicoanálisis destaca el proceso de construcción de la identidad como parte de las tareas adolescentes en relación con el medio que le rodea, a la vez que legitima la importancia de elaborar psicológicamente lo que implica dejar las etapas anteriores del ser humano. Más adelante se profundizará de manera específica en este tema.

Para mostrar el énfasis en lo social, la investigadora Krauskopf es un buen ejemplo, pues plantea que la adolescencia «es el periodo en que se produce con mayor intensidad la interacción entre las tendencias individuales, las adquisiciones psicosociales, las metas socialmente disponibles, las fortalezas y desventajas del entorno» (Krauskopf, 2003:7).

Sin desdeñar la riqueza que existe en las definiciones consultadas, preferimos asumir básicamente el concepto que señala la adolescencia como «un período de desarrollo del ser humano mediante el cual se consolida una serie de cambios o integraciones biopsicosociales suficientes y necesarias como para que el niño se transforme y asuma el papel de adulto integrado a la sociedad» (Machado, 2001:15).

El término básicamente se refiere a que si bien esta definición sintetiza en pocas palabras tres elementos que poseen total relevancia - comprensión de la adolescencia como etapa de desarrollo, integraciones desde lo social, lo psicológico y lo biológico, ${ }^{1}$ y la transformación del niño en adulto con capacidad de inserción social—; obvia otros tres aspectos muy importantes: límite etáreo, las adquisiciones y desprendimientos como parte esencial de las integraciones sociopsicobio y el logro de niveles importantes de autonomía como meta final. Los matices que brinda la inclusión de ellos, permitirían un alcance conceptual mayor, para definir y comprender la adolescencia.

\section{CONCEPTO DE ADOLESCENCIA. PROFUNDIZACIÓN EN SUS PRINCIPALES ELEMENTOS}

El análisis anterior nos brinda la posibilidad de reconceptualizar el término adolescencia y asumirla como un periodo de desarrollo del ser humano, que sin poder enmarcarse en límites etáreos precisos, está comprendido entre las edades de 10 y 19 años aproximadamente. En él se consolidan una serie de cambios e integraciones desde lo social, lo psicológico y lo biológico (sociopsicobio); que sobre la base de adquisiciones y desprendimientos cualicuantitativos, permiten el alcance de

1 Aunque el concepto refiere biopsicosociales, se modifica el orden en correspondencia con lo ya planteado al respecto. 
suficientes y necesarios niveles de autonomía, para que el niño se transforme y asuma el papel de adulto integrado a la sociedad.

Si bien ninguna conceptualización abarca todos los elementos del objeto al cual se refiere, a nuestro juicio, la anteriormente expresada incluye aspectos fundamentales y es coherente con los presupuestos teóricos que compartimos. Integra diversas perspectivas que a continuación serán esbozadas, tras la idea de que ahí precisamente, y no tanto en una síntesis conceptual, puede emerger una mayor comprensión en torno al periodo.

La noción de adolescencia como periodo de desarrollo no ha existido siempre. Durante la comunidad primitiva, se entendía que era «un momento representado por un ritual de paso de una etapa de la vida a otra en la cual se accede a la sexualidad activa, se adquieren responsabilidades y poder dentro de la tribu» (Mead, en Obiols, 1995:39). Más recientemente en la historia, la autora Bozhovich, se refirió a ella como una «edad de transición, ya que es precisamente en este periodo en que se da un paso decisivo en el desarrollo del niño, al concluir su infancia y pasar a la etapa del desarrollo psíquico que lo prepara directamente para la vida laboral independiente [...] tiende hacia el futuro y todo su desarrollo comienza a determinarse por esta tendencia» (Bozhovich, 1976:195).

En ambos casos, aparece la idea de transición. De acuerdo con la forma en que se entienda el término puede resultar contrario al desarrollo, pues parecería que transitorio al fin, es un periodo de latencia, pasajero, del cual no hay que preocuparse. Por supuesto, esta mirada traería grandes peligros considerando las importantes transformaciones que se dan en las edades comprendidas en la etapa adolescente y su papel en el desarrollo ulterior de los seres humanos. La investigadora Krauskopf describe y critica el paradigma tradicional, que concibe la adolescencia como un período de preparación, durante el cual los sujetos son niños grandes o adultos en formación. Desde este punto de vista se expropia a la etapa de contenido intrínseco e «implícitamente se les niega el reconocimiento como sujetos sociales. A partir de ello se prolonga la dependencia infantil, se limita la participación y se genera la distinción/oposición entre menores» (Krauskopf, 2003:14).

En esencia, es por esta razón que compartimos el término transitorio desde la perspectiva contemporánea que ofrece el investigador Dávila, y su equipo de trabajo (2008). Para ellos, a partir de la interpretación que realizan de otros autores, las transformaciones en los modelos y procesos de entrada a la vida adulta llevan a entender la 
adolescencia como etapa de transición; lo cual delimita una situación de dependencia hacia una de mayor independencia. Los límites temporales en los que se enmarca el tránsito, se han modificado, según ellos, en la misma medida que lo han hecho las características de los espacios de inserción social de adolescentes y jóvenes (escuela, mercado laboral).

Estas ideas adquieren particular relevancia, cuando se añade la diferencia presentada por Dávila, entre transición considerada movimiento (la trayectoria biográfica que va de la infancia a la edad adulta) y transición asumida como proceso (de reproducción social). Para este último caso, el tránsito va más allá de historias vitales personales, pues más bien constituye un reflejo de las estructuras y los procesos sociales. Se destacan entonces los «procesos a nivel de la configuración y percepciones desde la propia individualidad y subjetividad del sujeto, y las relaciones que se establecen entre aquéllas y los contextos a nivel de las estructuras sociales en las cuales se desarrollan aquellas subjetividades» (Dávila et al., 2008:57). Esta realidad teórica llevada a sujetos concretos de la vida cotidiana, muestra que transitar por la adolescencia supone una compleja integración de lo individual con lo social, de ahí que el concepto propuesto considere esencial explicitarla y legitime la noción de adolescencia como etapa de desarrollo.

Si bien ya se comentó acerca de las disquisiciones respecto a los límites etáreos, vale la pena detenernos en las principales transformaciones que tienen lugar como integraciones desde lo social, lo psicológico y lo biológico. Probablemente sea éste uno de los temas más sistematizados y la literatura consultada no recoge discrepancias importantes asociadas a ellos.

Psicológicamente, los principales contenidos de la personalidad comienzan a esbozar las características particulares del futuro adulto. Surge el pensamiento teórico que les permite realizar reflexiones más profundas, elaborar hipótesis con juicios enunciados verbalmente y comprobables a través de un proceso deductivo, de ahí que llegar a generalizaciones con mayor amplitud favorezca la crítica ante su realidad y el ofrecimiento de argumentos sólidos para avalar sus acciones. Estos cambios cualitativos permiten que el adolescente sea capaz de adecuarse a las características que adquiere el estudio, pues varía el contenido y el método de obtención de conocimiento. Ello influye en el desarrollo de intereses cognoscitivos y profesionales.

La esfera afectivo/motivacional comienza a caracterizarse por gran excitabilidad, los sentimientos se hacen más variados y profun- 
dos, además de desarrollarse uno cualitativamente diferente: el amoroso. Aparece un conjunto de puntos de vista, juicios y opiniones propias de carácter moral, que participan en la regulación de su conducta con relativa independencia de la situación misma. Se amplía la gama de actividades que motivan a la persona y por tanto la jerarquización se convierte en un aspecto importante para guiar la conducta, en este caso con mayor libertad que en etapas anteriores. Todas estas transformaciones enunciadas grosso modo, se estructuran en un proceso que lleva implícito contradicciones y pérdidas, además de las adquisiciones fácilmente visibles en lo descrito.

Respecto a lo biológico, llaman la atención las visibles modificaciones físicas en un periodo relativamente corto de tiempo. El «bullicio hormonal», como se le ha llamado a lo que ocurre desde el punto de vista endocrino, tiene su expresión en las dimensiones físicas del cuerpo (crecimiento de todas las estructuras e incremento de la masa corporal principalmente), en la fisiología del organismo (deficiencias del sistema circulatorio, desórdenes en el funcionamiento del sistema nervioso) y en la maduración sexual (aparición de los caracteres sexuales secundarios y cambios en los primarios, aparición de la primera eyaculación y la primera menstruación, etc).

Finalmente, entre los elementos que aparecen en el concepto propuesto, el alcanzar niveles de autonomía que posibiliten la «conexión saludable» del joven/adulto con su entorno, sería el fin último de la adolescencia. Aunque parecería ésta una obviedad, ni el desarrollo de las ciencias ha dado suficientes evidencias de que este hecho se haya considerado como tal, ni las condiciones en que actualmente tiene lugar el crecimiento humano parece estar en función de ese resultado. Por lo menos cabría preguntarse qué significa crecer en la adolescencia hoy, y cómo se conecta este proceso con el sistema de relaciones en que están inmersos los sujetos, llegado ese momento de la vida. Estas interrogantes nos permitirán acercarnos a las transformaciones que desde lo social tienen lugar en la adolescencia, así como, harán posible esbozar algunas contemporáneas realidades al respecto.

\section{CRECER EN LA ADOLESCENCIA HOY. UNA MIRADA AL SISTEMA DE RELACIONES QUE SE ESTABLECEN EN LA ETAPA}

Crecer es un término muy utilizado en el discurso científico y en el lenguaje común cuando de seres vivos se trata; sin embargo, plantea condicionantes y cualidades diferentes según sea el caso. Por esa ra- 
zón, no son idénticas referencias si del tema habla un biólogo, unos padres angustiados por sus hijos o un psicólogo. Para el caso que nos ocupa, el análisis se enmarca en la subjetividad, está muy asociado al desarrollo psíquico de los seres humanos, y aunque recibe la influencia del incremento etáreo y del crecimiento biológico, tiene sus especificidades.

Entre las distintas corrientes psicológicas que se han acercado al tema, los postulados de la psicología soviética se destacan por la comprensión que les permitió hacer la filosofía marxista y la concepción dialéctica de los fenómenos. Autores como Bozhovich (1976), Elkonin (Elkonin, en Colectivo de Autores, 2001a) y Leontiev (Leontiev, en Colectivo de Autores, 2001a), muestran en sus trabajos análisis importantes al respecto. En general, estos investigadores comparten la idea vigotskiana de concebir el desarrollo como «el proceso ininterrumpido de automovimiento que se caracteriza, en primer lugar, por el constante surgimiento y la formación de algo nuevo, inexistente en los estadios anteriores» (Vigotsky, 1987:15).

Este concepto estuvo acompañado de otros como periodos líticos, crisis evolutiva de la edad, neoformaciones y Situación Social del Desarrollo (SSD). Con ellos, se declara la presencia de momentos estables en el desarrollo psíquico, acompañados de momentos en los que se evidencia con claridad la aparición de nuevas cualidades (neoformaciones) que, al entrar en conflicto con las existentes y tras un periodo de rupturas imprescindibles, logran imponerse resultando una etapa superior del desarrollo. Asimismo, la SSD aporta la combinación entre las estructuras internas del sujeto y las condiciones externas en las que está viviendo, con lo cual queda planteada la coherencia con una ideología que ubica a lo social como una condicionante importante del desarrollo.

Debe distinguirse que los términos desarrollo y crecimiento no hacen alusión específicamente al mismo fenómeno, pues este último concibió la necesaria autonomía respecto al imaginario social instituido (Cucco, 2006), lo cual no fue comprendido por la psicología soviética. Además, y a criterio Segarte, el crecer trabaja más el problema del sujeto histórico para develar aquello que lo ata al instituyente; y en esa misma medida profundiza en cómo «zafarlo».

De cualquier modo, los estudios llevados a cabo en la ex Unión Soviética fueron numerosos, y debe destacarse la singular particularidad adjudicada a la actividad y la comunicación en cada periodo etáreo. En este caso, no son simples categorías a lo largo de la existencia humana, sino que se conjugan de forma tal, que a cada etapa corres- 
ponde una actividad rectora y a ésta, un modo de comunicación que permita a los individuos situarse en posición de pasar al estadio superior. Para los defensores del enfoque histórico-cultural, «la actividad no es respuesta o reflejo solamente, sino que implicaba un componente de transformación del medio con ayuda de instrumentos» (Rivière, 1988:41); mientras que la comunicación constituye un medio para realizar los distintos tipos de actividad.

En el caso específico de la adolescencia, la actividad rectora es la relación íntimo-personal (en su etapa temprana), mientras que con el avance de la edad va adquiriendo mayor relevancia la orientación científico-personal (Elkonin, en Cruz y Kraftchenko, 1983). Esta actividad ubica al adolescente en un espacio diferente al que tenía como escolar, por tanto, su posición social varía, se amplía el espectro de relaciones del individuo, y la comunicación con los otros se transforma. Comienzan así, a ponerse en práctica diferentes mecanismos de intercambio con los coetáneos (entiéndase amigos y pareja).

Los aportes de esta concepción son innegables, pues desde los primeros postulados de Vigotsky, la psicología dio un viraje sustancial en torno a la comprensión del ser humano. El enfoque históricocultural y el papel de lo social en la construcción de la subjetividad en desarrollo, fue novedoso. Sin embargo, el marcado énfasis que se hace en las adquisiciones a lo largo del desarrollo (neoformaciones) en contraste con el papel de los desprendimientos que en cada etapa deben ocurrir, constituye una limitación importante.

Por tal razón, consideramos imprescindible reconocer la dinámica que ofrecen al proceso de crecer, tres organizadores del psiquismo que - desde un psicoanálisis no ortodoxo- permiten la adaptación activa del ser humano al medio social. Asumirlos supone que las experiencias en las diferentes etapas son elaboradas de manera que el tránsito por cada una de ellas implica un grupo importante de adquisiciones; y a la vez, brinda igual importancia a los desprendimientos que también influirán para alcanzar el estadio superior.

El primero de ellos es el narcisismo, el cual resulta vital en los primeros momentos de la vida y supone la capacidad de quererse y a la vez reconocer que se es querido por otros. Ofrece la seguridad básica que todo ser humano necesita como contención psicológica esencial para asumir las experiencias de su existencia.

En segundo lugar está el duelo. En este caso se refiere a la necesaria elaboración de las pérdidas materiales o espirituales, cuyo papel se inicia cuando el bebé comienza a elaborar la pérdida que significa 
no estar siempre junto a mamá, al enfrentarse a no tener una satisfacción inmediata de las necesidades $\mathrm{y}$, a partir de tener experiencias que le ayuden a ubicar lo más saludablemente posible las vivencias que va teniendo como ser independiente.

En tercer lugar, la triangulación es el organizador del psiquismo relacionado con la aceptación de la exclusión respecto a un tercero. Inicialmente ocurre en la relación madre/hijo por la presencia del padre, luego con la aceptación de que existen espacios donde ambos progenitores comparten y en los que el hijo no puede estar, y a continuación en la adolescencia, con la búsqueda de nuevos lugares en el «adentro» (con los padres) y en el «afuera» a partir de los nuevos vínculos.

Aunque existen períodos en los cuales un organizador es más importante que otro y constituye un suceso medular para las adquisiciones y los desprendimientos que definen el proceso de crecer, nuestra dinámica psicológica los integra de forma tal que nos triangulamos en la medida que logramos hacer mejores duelos y ello es posible en tanto estamos bien narcisizados.

De esta forma, el proceso de crecer toma en consideración esencialmente la necesidad psicológica de la persona y a ello debe responder el entorno social en el cual el individuo se encuentre. Además, debe culminar en el alcance de la autonomía, que en palabras del científico griego Cornelius Castoriadis es una condición a la cual los sujetos han llegado cuando pueden «reflexionar sobre sí y su sociedad, cuando tienen un nosotros, e instituye — con conocimiento - un campo de significaciones imaginarias sociales (es decir, tienen una relación lúcida con éstas), reconociéndose como creadores» (Castoriadis, en Cucco, 2006:73).

Si bien podrían ser más amplias las referencias respecto al proceso de crecer, los elementos hasta aquí planteados permiten un bagaje teórico importante en aras de entender las condiciones en las que transcurre la adolescencia en la sociedad actual (nivel macro); las características de la comunicación en cada uno de los grupos a los cuales pertenecen los y las adolescentes; y las necesidades a las cuales responde dicha comunicación (nivel meso en ambos casos) y qué impacto tiene todo eso en la construcción del ser humano (nivel individual). Al respecto, varios autores han referido en sus análisis, la impronta que va dejando la postmodernidad en la formación de los adolescentes durante los últimos años. Entre ellos pueden mencionarse Obiols y Di Segni (1995), Krauskopf (2003), Cucco (2006) y Dávila et al. (2008).

Específicamente Cucco, sintetiza lo que a su juicio constituyen indicadores de la cultura postmoderna que inciden directamente en la articu- 
lación de la subjetividad de los adolescentes. En este sentido menciona el cuestionamiento a la pretensión de las ciencias de llegar a verdades absolutas y universales; la apología de las diferencias que esconde la propuesta del más brutal individualismo y la impunidad de su desarrollo y hegemonía; el rescate de valores hedonistas con la consiguiente necesidad de satisfacer inmediatamente los deseos; el planteamiento de una ruptura de la temporalidad, es decir, «el ya fue» de la experiencia recién vivida; el vaciamiento de la palabra, imponiéndose la imagen; y el ofrecimiento de «bienestar» que lleva inevitablemente al vacío (Cucco, 2006).

De acuerdo con la interpretación a la que es factible llegar partiendo del análisis realizado por la $\mathrm{Cucco}$, dichos indicadores promueven una cultura del «todo vale», que no permite la crítica de la vida cotidiana y sitúa al adolescente en una posición de poca contención, cuando numerosas transformaciones están ocurriendo en su interior. A la vez, le impiden hallar un lugar en la sociedad desde las redes sociales más cercanas; dificulta sus vínculos al no tener capacidad de espera para satisfacer sus deseos y no considerar al otro; coloca al adolescente en una «atemporalidad» que imposibilita la resolución de su proceso de maduración y dificulta sustancialmente la elaboración de un proyecto de vida más o menos claro.

En este sentido, Krauskopf plantea que «el futuro como meta orientadora se ha tornado incierto por la velocidad de las reestructuraciones sociales y culturales [...], se avizora desde las perspectivas que brinde el presente y se construye a partir de aproximaciones sucesivas y diversas» (Krauskopf, 2003:8). Similar mirada le dan al fenómeno Dávila y sus colegas (2008) al analizar las particularidades de las trayectorias juveniles en la actualidad y la imposibilidad de vivirlas como lineales, partiendo de las incertidumbres que se muestran en las sociedades actuales. Ante tal panorama macrosocial: ¿cómo se instrumenta el sistema de relaciones de los y las adolescentes?

Previo al análisis concreto, debe recordarse, que las transformaciones en la posición social del adolescente son vitales para su desarrollo. En este sentido, la familia, se convierte en un ámbito que evidencia la necesidad de modificar los estilos de relación entre ellos y los adultos. El sistema de obediencia y subordinación característico de otras etapas se hace inadmisible, de ahí que la comunicación comience a exigir matices cualitativamente diferentes y necesite nuevas formas de expresión. Paralelamente a ello, las reestructuraciones que sufre la identidad adolescente, demandan tiempos de intimidad consigo mismo, lo cual deja «excluida» a la familia. 
El o la adolescente comienza a construir una nueva percepción de su mamá y su papá, experimentando una clara desidealización. La imagen de adultos omnisapientes y todopoderosos, es remplazada por otra mucho más realista. La ampliación de las relaciones a grupos de amistades, así como las parejas amorosas tan importantes en este período, genera una reubicación obligada de la familia, para lo cual esta última, en ocasiones no está preparada.

Todo ello propicia la búsqueda de un espacio en el afuera, ${ }^{2}$ por parte del o la adolescente. Así, con las contradicciones de un proceso que está teniendo lugar, el grupo primario debe abrir sus límites y comenzar el adiós necesario hacia la mayor independencia del individuo, pues las transformaciones antes mencionadas ocurren en función de una nueva posición social para quien se encuentra en la adolescencia.

Sin embargo, he intentando responder la interrogante planteada en torno a la instrumentación del sistema de relaciones de los y las adolescentes, puede decirse que es frecuente ver padres sin reales modelos de autoridad, estableciendo relaciones de paridad con los hijos, sobrecargados por la ausencia de límites en las pautas de crianza; con conflictos por la propia concepción de lo que es ser padres y la separación de los hijos, por la asimilación de un tipo de pautas de crianza poco saludables, y en consecuencia, atormentados por la falta de recursos para decodificar las necesidades del proceso de crecimiento de sus hijos y potenciar la maduración y la autonomía (Cucco, 2006). De hecho, «la familia ha visto seriamente afectada su labor principal de contribuir al desarrollo de los procesos básicos de autonomía. Muy por el contrario ha sido signada para recrear múltiples procesos de dependencia, distorsionadores de un saludable proceso de crecimiento» (Cucco, 2006b:4).

Paralelamente a esta dinámica, en la ampliación de las relaciones, «esperan» al adolescente, los coetáneos y la pareja. Con los primeros constituye un espacio de construcción psicológica muy importante en el cual se tienen experiencias de posicionamiento social fuera de la familia. Esto hace que la necesidad de reconocimiento grupal regule la conducta y los interlocutores se conviertan en guías del comportamiento. Al inicio se relacionan con los pares del mismo sexo y luego comienzan a integrarse más. En general, aunque el proceso no se da tan lineal, ni la influencia familiar desaparece, el poder que logra tener un grupo de adolescentes sobre sus miembros es alto (Kon, en Domínguez, 2003; Oliva, en Domínguez, 2003).

2 Se refiere a los vínculos fuera del entorno familiar. 
Surge entonces una situación en la que se anhelan algunas circunstancias de la infancia, pero se desean las propuestas del nuevo mundo que se abre camino. En tales casos, la familia debe ser más flexible ante las experiencias que propone la adolescencia, sin concebir antagonismos per se en las nuevas relaciones. Por el contrario, la posibilidad de establecer límites educativos en total correspondencia con el periodo, y sobre todo, tener claridad de cuál es el camino mejor para que sean jóvenes y adultos psicológicamente fortalecidos y saludables, constituye un modo de acompañar a los adolescentes sin interrumpir su normal dinámica de crecimiento.

Así mismo, las relaciones amorosas y la práctica activa de la sexualidad se conjugan para darle definitivo matiz diferente al sistema de relaciones que establecen los adolescentes. Probablemente, por primera vez en la vida se enfrentan a necesidades y situaciones importantes, cruciales, sin la presencia de la mamá y el papá. En este espacio se reafirma también su identidad, adquieren una expresión particular las concepciones que sobre los roles de género se tengan y tras intercambios de pareja, comienzan a conformarse las bases de lo que será la futura familia. A este contexto, como al de los amigos, es muy importante que el adolescente llegue psicológicamente preparado; sin embargo, la educación actual no propicia de forma suficiente una estructuración psicológica que ayude al establecimiento de relaciones sanas y desarrolladoras.

No es sólo la familia la que en muchos casos no se encuentra preparada para ofrecer las alternativas de relación que posibiliten el mejor crecimiento; sino que otros espacios también basan sus pautas educativas en postulados extemporáneos, perpetuadores de estereotipos, centrados en el control de la conducta más que en modificaciones de esencias, guiados por ambivalencias en las pautas educativas y a través de métodos sutiles muy difíciles de develar.

Parecería que algún «plan macabro» se ha diseñado para esbozar estas realidades; sin embargo, si así fuera, la tarea de reconstrucción se presentara más sencilla o al menos exhibiera mayor claridad. En nuestra opinión, el entramado de circunstancias que ha ido conformando esta realidad es sumamente complejo y sólo puede comprenderse a cabalidad adentrándose en la relación sociedad, grupo e individuo; profundizando en sus dinámicas actuales y asumiendo con total responsabilidad el costo que implica romper con lo dañino que está instituido y legitimar nuevos y más saludables instituyentes.

Esta sucinta mirada a la adolescencia no refleja todos los matices posibles a observar en nuestra vida cotidiana. Sin embargo, alerta 
sobre los caminos que va teniendo un proceso de socialización con total potencialidad para ser enriquecedor, pero con contantes amenazas de daño. Las estructuras sociales, las premisas de vida que guían nuestra existencia y el papel de los diferentes espacios de socialización constituyen elementos cruciales para que algo podamos hacer. Debemos evitar la proliferación de fenómenos socialmente perjudiciales, debemos trabajar para que no continúen insertándose las «enfermedades» de la macroestructura, en los espacios de formación de los individuos y facilitar así, una construcción de la subjetividad más coherente con lo que significa ser humano y vivir en sociedad. Corresponde entonces a las Ciencias Sociales brindar su granito de arena y poner a disposición reflexiones y modos de actuación social que colaboren con este empeño.

LA HABANA (CUBA), SEPTIEMBRE 2009

RECIBIDO: SEPTIEMBRE 2009

ACEPTADO: OCTUBRE 2009

\section{REFERENCIAS BIBLIOGRÁFICAS}

BOZHOVICH, L. I. (1976): La personalidad y su formación en la edad infantil. La Habana: Editorial Pueblo y Educación.

Colectivo De Autores (2001): Psicología del desarrollo. Selección de lecturas. La Habana: Editorial Félix Varela.

CucCo GarcíA, MiRTHA (2006a): ProcC: una propuesta de intervención sobre los malestares de la vida cotidiana. Buenos Aires: Editorial Atuel.

- (2006b): «Aspectos psicosociales de la adolescencia». En Primer Taller de Coordinadores de Grupo Formativo. La Habana: Editorial Cenesex.

DÁvila León, OsCar; Felipe GHIARdo y Carlos Medrano (2008): Los desheredados. Trayectorias de vida y nuevas condiciones juveniles. Valparaíso: Ediciones CIDPA (cuarta edición).

Domínguez García, Laura (2003): Psicología del desarrollo: adolescencia y juventud. La Habana: Editorial Félix Varela.

ELKONIN, D. B. (1983): «Acerca del problema de la periodización del desarrollo psíquico en la edad infantil». LEYDA CRUZ y OKSANA KRAFTCHENKO (compiladoras): Selección de lecturas de psicología infantil y del adolescente. Primera parte. La Habana: Ediciones Unión.

GutiÉRrez BARÓ, ElSA (2007): «Adolescencia y juventud. Concepto y características». Disponible en: www.psicolt.salud/c/html. 
KNoBel, M. (2008): «El síndrome de la adolescencia normal». Disponible en: www.psicología.chl.

KRAUSKOPF, DINA (2003): Participación social y desarrollo en la adolescencia. San José: Fondo de Población de las Naciones Unidas.

MARTíneZ ZARANDONA, IRENE (2005): «El concepto de adolescencia». Disponible en: http://sepiensa.org.mx.

MACHADO MEDINA, ADILÉN (2001): La violencia intrafamiliar en relación al rol de género. Percepción de un grupo de adolescentes. La Habana. Tesis de Maestría, Centro Nacional de Educación Sexual.

Obiols, GuILlermo y Silvia Di Segni (1995): Adolescencia, posmodernidad y escuela secundaria. La crisis de la enseñanza media. Buenos Aires: Kapelusz.

OPS/OMS (2002): Informe mundial sobre la violencia y la salud: resumen. Washington: OPS/OMS.

RIVIÈRE, ÁNGEL (1988): La psicología de Vygotski. Madrid: Gráficas Valencia.

VIGOTSKY, L. S. (1987): Historia del desarrollo de las funciones psíquicas superiores. La Habana: Editorial Científico-Técnica. 